\title{
Practical Broad-Band Tuning of Dye Lasers by Solvent Shifting
}

\author{
SUSAN M. GRENCI, $\dagger$ GEORGE R. BIRD, $\ddagger$ BRIAN W. KEELAN \\ and AHMED H. ZEWAIL§
}

Arthur Amos Noyes Laboratory of Chemical Physics, || California Institute of Technology, Pasadena, California 91125

(Received July 26, 1985)

\begin{abstract}
We have operated a dye laser over a broad wavelength range $(593.8-667.0 \mathrm{~nm})$ by shifting the dye emission profile with incremental changes of solvent composition. This was accomplished with the laser operating continuously, and only minor adjustment of the laser optics was required. Solvent tuning was facilitated by the critical dependence of the optimum laser wavelength on concentration of the second solvent. Using the known solvent-sensitive laser dye 9-diethylaminobenzo[a]phenoxaz-5-one (DBP), 87\% of the tuning range from pure xylenes to pure methanol was covered by cumulative addition of $24 \mathrm{vol}$. \% methanol to the starting xylenes solution. The optimum dye concentration was found to be independent of solvent composition, so that maximum laser power could be maintained by mixing equimolar dye solutions in the two solvents. These results establish the practicality of solvent-tuning as a method of conducting laser experiments over a broad wavelength range.
\end{abstract}

KEY WORDS: Dye lasers; Solvent shifts of dye emission.

\section{INTRODUCTION}

In this paper we explore the practicality of tuning a dye laser over a broad range of wavelengths by incrementally varying solvent composition. This method has been suggested earlier by the Göttingen group, and Halstead and Reeves ${ }^{2}$ have optimized a coumarin dye for emission

† Work by S.M.G. described herein completed at Rutgers University; present address: E.I. DuPont Co., Photoproducts Division, P.O. Box 267, Brevard, North Carolina 28712.

¥ Visiting Professor at California Institute of Technology; permanent address: Department of Chemistry, Rutgers University, New Brunswick, New Jersey 08903; author for correspondence.

$\S$ Camille and Henry Dreyfus Teacher-Scholar.

$\|$ Contribution \#7258. 
at $507 \mathrm{~nm}$ with a selected mixture of dioxane-ethanol. The latter workers determined the dependence of the peak emission wavelength on solvent composition at fixed dye concentration. Ethers, such as dioxane, have been avoided in this study, both because of their low flash points and their tendency to combine with ambient oxygen to form reactive peroxides.

The Göttingen group investigated DBP as a laser material in several solvents and called attention to the possibility of shifting the dye with solvent mixtures. They obtained lasing action from 580 to $631 \mathrm{~nm}$ in diethyl ether, from 690 to $743 \mathrm{~nm}$ in hexafluoroisopropanol, and over the entire range from 580 to $743 \mathrm{~nm}$ in these and intermediate solvents. Using a long duration flash lamp $(15 \mu \mathrm{sec})$ they found comparable output powers from DBP and Rhodamine $6 \mathrm{G}$ in ethanol for pumping well above threshold, though the DBP threshold was $45 \%$ higher than that of Rhodamine $6 \mathrm{G}$. We have found DBP to be significantly less efficient than Rhodamine $6 \mathrm{G}$ in our apparatus; the difference between $6 \mathrm{nsec}$ and $15 \mu \mathrm{sec}$ laser pumping is probably due to triplet quenching, as discussed below.

We have obtained efficiency curves (photon flux vs. wavelength) for DBP in various mixtures of methanol and xylenes. Several results substantiate the practicality of solvent-tuning. First, most of the change in the emission profile between xylenes and methanol occurs upon addition of small quantities of methanol to xylenes. Thus the laser may be operated continuously during this procedure, and minimal realignment of dye laser optics is required as the index of refraction of the dye solution changes. Second, the optimal dye concentration is invariant with respect to solvent composition. Consequently, the concentrations do not have to be reoptimized after second solvent additions if the second solvent contains dye in the same concentration as the first.

\section{EXPERIMENTAL}

The laser apparatus consisted of a scanning dye laser (Quanta-Ray PDL-1) pumped by a KDP frequency-doubled Nd:YAG laser (Quanta-Ray DCR-1, oscillator only). The green $(532 \mathrm{~nm})$ pump characteristics were: pulse energy $53 \mathrm{~mJ}$, duration $6 \mathrm{nsec}$, and repetition rate $10 \mathrm{~Hz}$. The pump power is divided among the oscillator, a 
preamplifier (optional), and an amplifier. The oscillator is sidepumped and is coupled to a telescope and diffraction grating. The diffraction grating, used in fourth order in these experiments, limits the oscillator cavity to a low $Q$ value.

The dye used in this experiment, DBP, is a phenoxazone closely related to the oxazine dye Nile Blue A (Colour Index 51180). DBP is not commercially available, although a source is erroneously given in Ref. 1. There would appear to be a conflict between the broad class patent issued to Drexhage in $1974^{3}$ and the more specific patent issued to Basting in $1976 .{ }^{4} \mathrm{DBP}$ was synthesized in a single step by hydrolyzing the $-\mathrm{NH}_{2}$ group of commercial grade Nile Blue in aqueous $\mathrm{HCl}$ and separating the neutral product dye following Thorpe. ${ }^{5}$ The reaction time was extended to maximize reaction yield. Agreement between elemental analysis $(\mathrm{C}=75.37 \%, \mathrm{H}=5.49 \%, \mathrm{~N}=8.55 \%)$ and calculated values for $\mathrm{C}_{20} \mathrm{H}_{18} \mathrm{~N}_{2} \mathrm{O}_{2}(\mathrm{C}=75.45 \%, \mathrm{H}=5.70 \%, \mathrm{~N}=$ $8.55 \%$ ) was excellent.

The solvent pair consisted of xylenes as the first solvent and spectroscopic grade methanol as the second solvent. Xylenes refers to an ACS reagent grade mixture of the three isomeric xylenes. It is used here as a benzene substitute, having lower toxicity and a higher flash point. These solvents were used without further purification.

In preparation for the use of unusual solvents such as xylenes, the $O$-rings in the dye laser were changed from the original copoly(ethylene-propylene) O-rings to Viton ${ }^{6}$ poly(1,1-difluoroethylene) O-rings. This material is resistant to many but not all organic solvents. Before using a new solvent in the dye laser circulating system, we recommend that a sample O-ring be weighed, immersed overnight in the trial solvent, blotted dry, and weighed again. We rejected dimethylformamide as a possible solvent after weight gains from $50 \%$ to $100 \%$ were obtained. The weight gain of a Viton O-ring in xylenes was less than $1 \%$, which we consider acceptable trace uptake by the material. The other potentially vulnerable material in the circulating system is a cellulosic filter for lint and bubble removal. This was resistant to xylenes and to methanol, but in chlorobenzene we have seen these filters swollen and loosened to the point of becoming a source of particulates.

While our solvent additions were accomplished by pipetting directly into the reservoirs, we would recommend a more convenient method of addition for routine usage. Two pairs of small and large hypodermic 
syringes $(10$ and $250 \mathrm{ml})$ with small bore teflon tubing and one of the old-fashioned hypodermic blood transfusion valves for each of the syringes would allow convenient additions to the oscillator and amplifier reservoirs during the course of the experiment.

The photon flux was measured by a Hammamatsu 1P28 photomultiplier after attenuation of the laser power by 6-7 orders of magnitude with neutral density filters, a pinhole, and a diffusing window. Linear operation of the detector was verified by inserting a calibrated neutral density filter in the optical train and confirming the appropriate decrease in signal. The detector sensitivity was compared with a calibrated silicon PIN photodiode from $580 \mathrm{~nm}$ to $680 \mathrm{~nm}$ using an auxiliary tungsten light source and a Jarrel-Ash $\mathrm{f} / 8$ monochromator. The photomultiplier calibration obtained was similar to that given by Hamamatsu but differed significantly from an S-5 calibration given by another manufacturer.

This calibration was used to generate output curves of relative photon flux vs. wavelength. To convert to a quantum efficiency scale, we measured the pump and laser output energies for the pure xylenes dye solution with a volume-absorbing calorimetric power meter (Scientech). This measurement was checked for consistency by measuring the efficiency of the laser with methanolic Rhodamine 6G. A value of $28 \%$ energy efficiency ( $30 \%$ quantum efficiency) was obtained for Rhodamine 6G, reasonable in light of the manufacturer's specification of $34 \%$ at somewhat higher pump power ( $70 \mathrm{vs.} 53 \mathrm{~mJ} /$ pulse).

\section{RESULTS}

The first dye solution to be tested in the laser was made to $500 \mu \mathrm{M}$ DBP in xylenes, based on an estimate from recommended concentrations (Quanta-Ray) for the rhodamine dyes which gave products of $\varepsilon C=25 / \mathrm{cm}$ at the pump wavelength $(532 \mathrm{~nm})$. Tests at other concentrations established an optimum of $530 \mu \mathrm{M}$ for DBP in xylenes in this oscillator. Use of the preamplifier resulted in lower output power, so it was removed from the optical train. End-pumping of the amplifier yielded higher output power than side-pumping. In this configuration, the optimum amplifier concentration was found to be $10 \%$ of the oscillator concentration. Under these optimized conditions, the energy 
efficiency of DBP was found to be $1.0 \%$ (quantum efficiency $1.1 \%$ ) at $612 \mathrm{~nm}$.

After plots of output flux had been obtained with the pure xylenes solutions in place, the volumes in the oscillator and amplifier solution reservoirs were reduced from one liter to $800 \mathrm{ml}$ to leave space for the addition of the methanol solutions. A preliminary examination of the absorption shifts on addition of $\mathrm{CH}_{3} \mathrm{OH}$ had indicated that much of the shifting would occur upon relatively small volume additions of $\mathrm{CH}_{3} \mathrm{OH}$. A series of successive additions of the methanolic solutions (with the same dye concentrations as the xylenes solutions) was made with volume increments of $1 \%, 1 \%, 3.13 \%, 6.25 \%$, and $12.5 \%$ for a cumulative volume addition of $23.8 \%$ methanol relative to the starting volume of xylenes. This was accomplished within the limits of the reservoir volumes between safe coverage of the intake tube and maximum reservoir capacity. It is especially important to note that all of these additions were made with the laser running, and that only minor retuning of the laser optics was necessary at the larger incremental additions to allow for the changes in refractive index of the solutions as methanol was added. After each addition and incidental alignment a curve of relative photon output flux was recorded as a function of wavelength.

The results of these additions and photon flux curves are given in Table I and shown in Figure 1. The efficiency curves for the second and fourth additions have been omitted from Figure 1 to avoid confusion, but these trials fall in continuity with the others, as may be seen in Table 1. Note that the limiting wavelength points have been determined by extrapolating the last linear portions of the efficiency curves. These points lie slightly inside the ultimate limit of lasing action.

The traces of half-maximum output points and limiting low power points are shown on a plot of wavelengths vs. mole fractions of methanol in Figure 2. This figure summarizes the practical use of this technique with DBP and this pair of solvents. It is preferable not to attempt laser experiments at wavelengths where power is very low and the laser is on the verge of going out of oscillation as a result of even a small change in gain. The track of the $50 \%$ maximum power points defines the preferred zone of operation. The results for pure methanol are shown on the right side of Figure 2 (note the broken scale of mole fractions, indicated by the jagged lines). The total range 


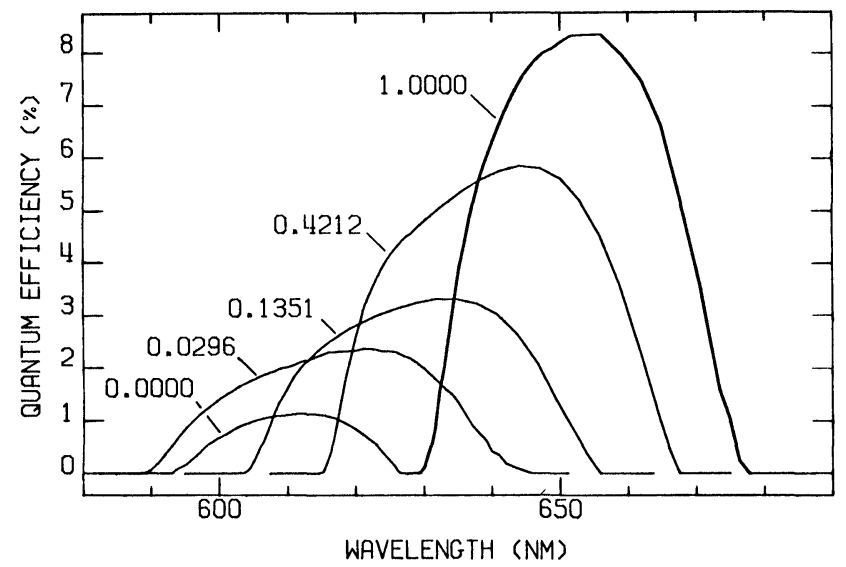

FIGURE 1 Quantum efficiency curves of DBP in different solvent mixtures. The number given for each curve is the mole fraction of methanol in each methanol-xylenes mixture. Nearly all of the tuning range from pure xylenes to pure methanol can be covered by cumulative addition of $24 \mathrm{vol} . \%$ methanol (mole fraction 0.4212 ).

of wavelengths available from pure xylenes to pure methanol is 669.6$599.2 \mathrm{~nm}=70.4 \mathrm{~nm}$ between lowest and highest half-power points. The solvent mixtures used in this experiment swept out a range of 660.3$599.2 \mathrm{~nm}=61.1 \mathrm{~nm}$ or $87 \%$ of the available range of this particular solvent pair. We regard this as a convenient way of securing a combina-

TABLE I

Performance characteristics of DBP in methanol-xylenes mixtures

\begin{tabular}{cccccccc}
\hline $\begin{array}{c}\text { Volume } \\
\text { percent } \\
\text { methanol }\end{array}$ & $\begin{array}{c}\text { Mole } \\
\text { fraction } \\
\text { methanol }\end{array}$ & $\lambda_{\text {lim }}^{-\mathrm{a}}$ & $\lambda_{1 / 2}^{-}$ & $\lambda_{\text {peak }}$ & $\lambda_{1 / 2}^{+}$ & $\lambda_{\text {lim }}^{+}$ & $\Phi_{\text {peak }}$ b \\
\hline 0.00 & 0.0000 & 593.8 & 599.2 & 612.5 & 622.3 & 626.3 & 1.1 \\
1.00 & 0.0296 & 5899 & 597.9 & 622.0 & 635.8 & 642.4 & 2.4 \\
2.00 & 0.0575 & 595.2 & 600.9 & 621.5 & 637.8 & 643.0 & 2.2 \\
5.13 & 0.1351 & 604.3 & 609.8 & 632.0 & 648.3 & 655.6 & 3.3 \\
11.38 & 0.2574 & 610.8 & 615.9 & 635.7 & 653.1 & 659.7 & 3.4 \\
23.88 & 0.4212 & 615.7 & 620.7 & 644.0 & 660.3 & 667.0 & 5.9 \\
- & 1.0000 & 630.4 & 635.6 & 654.0 & 669.6 & 674.8 & 8.3 \\
\hline
\end{tabular}

${ }^{a} \lambda_{\mathrm{lim} / \mathrm{half}}^{-/+}$refers to the lower/upper wavelength in $\mathrm{nm}$ corresponding to the limiting/half-power points of the efficiency curves. Limit points were estimated by extrapolating the linear portions of the efficiency curves and lie slightly inside the ultimate lasing range.

${ }^{b}$ Peak quantum \% efficiency (photons out/100 pump photons). 


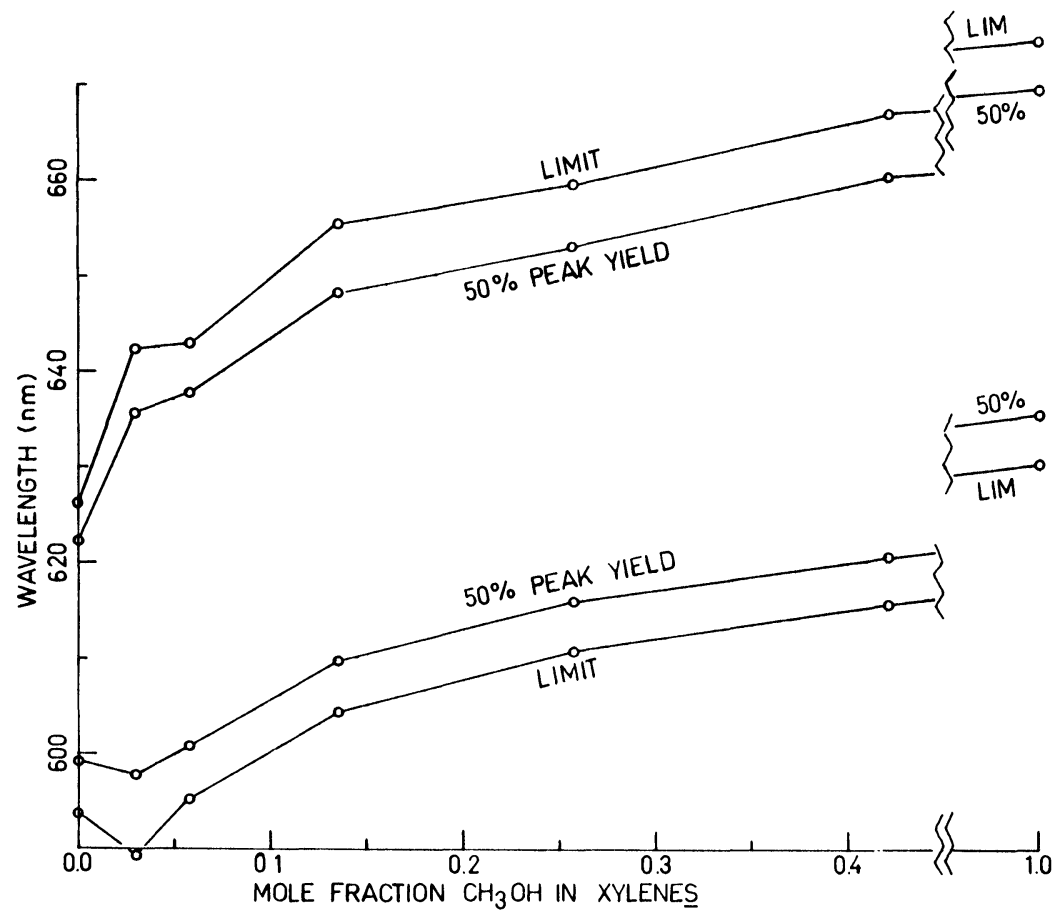

FIGURE 2 Variation of lasing limits and half-power wavelengths as a function of composition of methanol-xylene mixtures. Limit values were extrapolated from the linear portions of the efficiency curves and lie slightly inside the ultimate lasing range. Note the break in the composition axis; data points to the left of the break were obtained in a single continuous experiment without interrupting laser operation.

tion of optimal power and wide band tuning in a continuous dye laser experiment.

\section{DISCUSSION}

It is important for practical solvent tuning that the spectral shift of the dye occurs continuously as solvent composition is changed, unlike the effect of chemical alteration of the chromophore (e.g., acid-base equilibrium). This method of tuning is peculiar to certain merocyanines and highly unsymmetrical cyanines. For example, symmetric cyclized cyanines and azacyanines (rhodamines and oxazines respectively) do 
not have large solvent shifts, while some uncharged but dipolar merocyanines (such as rhodamones, cyclized coumarins, and DBP, a cyclized azamerocyanine) show considerable solvent sensitivity. ${ }^{7}$ The versatility of the large class of cyclized merocyanines and azamerocyanines has been demonstrated in earlier investigations of their lasing properties ${ }^{7-10}$ and their action in solid state photovoltaic cells. ${ }^{11,12}$

The solvent shifts of a number of uncyclized merocyanines, some of which are quite large, have been explored by Brooker and coworkers ${ }^{13}$ at the Kodak Laboratories. J. R. Platt ${ }^{14,15}$ has developed a phenomenological theory in which the spectral shift is a function of the chromophore chain length and the differing basicities (electron accepting tendencies) of two end groups. When the basicities of the two end groups are changed unequally by a new solvent, the dye is described as solvent-sensitive or "solvatochromic".

The solvent-shifting of DBP can be understood in terms of the two resonance structures depicted in Figure 3. In the absence of hydrogenbond donating solvents such as methanol, the left-hand nonpolar structure predominates in the ground state $S_{0}$. In the first excited state $S_{1}$ the importances of the two resonance structures are reversed and the dipolar structure predominates. Under these conditions the absorption band is relatively broad, the absorption maximum falls at a relatively short wavelength, and there is a large Stokes shift between the absorption and fluorescence peaks. This large Stokes shift is advantageous for good lasing performance because the strongest part of the emission band is available for laser action. In contrast, the rhodamine dyes lase out of a weak shoulder on the long wavelength edge of the fluorescence emission band. ${ }^{16}$

The behavior of DBP is quite different in water; the two resonance structures are of nearly equal importance in both $S_{0}$ and $S_{1}$. In this

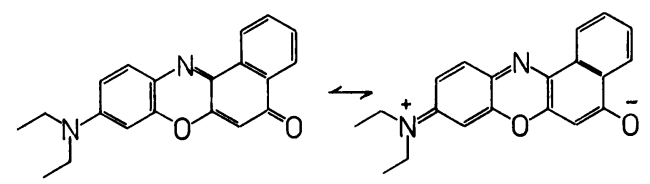

FIGURE 3 Resonance structures of the laser dye DBP. The right-hand structure makes only a minor contribution to $S_{0}$ in xylenes, but becomes more important in hydrogen-bond donating media such as methanol. 
case, very little change in the equilibrium nuclear coordinates occurs in the $S_{0} \rightarrow S_{1}$ transition, and the sharp absorption and fluorescence bands are nearly "mirror images". This is the isobasic or isoenergetic condition with minimal Stokes shift, and the spectrum of the dye mimics a symmetrical cyanine or rhodamine dye. It takes no subtle instrumentation to observe this kind of spectral shifting; the dye is orange in xylenes and lavender (almost blue) in water. Unfortunately, the solubility of DBP in water is too low to be practical for lasing.

One of us (S.M.G.) studied the absorption, fluorescence, and fluorescence quantum yield of DBP in a variety of solvents. ${ }^{17}$ The dye has a quantum yield of 0.89 in nonpolar xylene and yields on the order of 0.9 in polar solvents incapable of hydrogen-bond donation. It has yields of 0.50 in $\mathrm{CH}_{3} \mathrm{OH}$ and 0.64 in $\mathrm{CH}_{3} \mathrm{OD}$, and an activation energy of $3.0 \mathrm{kcal}$ for competitive fluorescence quenching in $\mathrm{CH}_{3} \mathrm{OH}$. These results were interpreted as indicating that hydrogen bond cleavage in the excited state opens a channel for internal conversion to the ground state. ${ }^{18}$ These results parallel those found for methyl salicylate in molecular beams (threshold energy $3.7 \mathrm{kcal}$ ) and in solution. ${ }^{19}$

The discrepancy between the high efficiency of DBP reported by Basting, Ouw, and Schäfer (power comparable to Rhodamine 6G in ethanol) and our lower efficiencies $(28 \%$ as quantum efficient as Rhodamine $6 \mathrm{G}$ in pure methanol) suggests that the long excitation pulse $(15 \mu \mathrm{sec})$ employed in their study favors DBP relative to Rhodamine $6 \mathrm{G}$. Rhodamine $6 \mathrm{G}$ does have an appreciable rate of intersystem crossing to the triplet state and does have a triplet-triplet absorption band which lies directly over the lasing region. ${ }^{20-22}$ Good performance is to be expected from Rhodamine $6 \mathrm{G}$ only at the start of the extended exciting pulse. In contrast, DBP may be giving moderately good performance over the full length of the extended exciting pulse. If so, we might explain the reduced triplet interference with DBP by some combination of a fast $T_{1} \rightarrow S_{0}$ reaction and a displacement of the $T_{1} \rightarrow T_{n}$ absorption away from the lasing region. We have seen that DBP in hydrogen bonding solvents does have a mechanism for rapid internal conversion $S_{1} \rightarrow S_{0}$ through the cleavage of $=\mathrm{O}-\mathrm{H}-\mathrm{O}-$ hydrogen bonds in the excited state, and this mechanism may also act rapidly to depopulate triplet states. This is suggested because the rising laser efficiency of DBP with added methanol is in contrast to the falling fluorescence quantum yield. There is always the possibility that triplet deactivation could be enhanced and also that 
excimer formation could be minimized through structural modification of the DBP molecule.

We should note that extreme solvent tuning may in some cases shift the absorption band of the dye away from the wavelength of excitation. Such a shift would require a change of concentrations to stay near optimum performance. Also note that having tuned by adding a volume of second solvent, it is not practical to attempt to reverse the direction of tuning by adding large volumes of the first solvent.

\section{CONCLUSION}

Two unexpected results of this study underscore the practicality of the method of solvent-tuning. The first of these is that with the dye and solvent pair studied the tuning is large and nonlinear upon addition of the second solvent. Thus a wide tuning range can be covered by the addition of a modest volume of the second solvent, so the laser can be operated continuously during the solvent changes. The second finding is that the optimum concentration of dye is insensitive to solvent composition, at least in the particular system we have examined. Thus, having found the optimum concentrations for the dye in the first solvent one can prepare dye at the same molar concentration in the second solvent so that mixing the two solutions leaves the dye concentration invariant. This greatly simplifies the mixing process and facilitates the method of solvent tuning.

The results of this work should encourage the application of solvent tuning to practical research. A variety of dyes and solvents may be amenable to this technique. For example, the solvent pair of xylenes and hexafluoroisopropanol (HFIP) may give a wider range of tuning with DBP than the solvent pair we have examined. The relatively new long wavelength dyes styryl-13 and styryl-14 are both highly unsymmetrical octamethine cyanines and should be subject to appreciable solvent tuning from the maxima at 925 and $980 \mathrm{~nm}$ respectively. ${ }^{23}$

\section{Acknowledgements}

We thank Larry W. Peng for experimental assistance. Financial support for this work was provided by the President's Fund of the California Institute of Technology, and by a National Science Foundation Grant to Rutgers University (CHE-791651). 


\section{References}

1. D. Basting, D. Ouw and F. P. Schäfer, Optics Comm. 18, 280 (1976).

2. J. A. Halstead and R. R. Reeves, Optics Comm. 27, 273 (1978).

3. K. H. Drexhage, U.S. Patent 3,731,222, 1 May 1983.

4. D. Basting, B.R.D. Patent 2,534,713, 17 Feb. 1977.

5. J. F. Thorpe, J. Chem. Soc. (London) 91, 324 (1907).

6. Trade name and product of the E.I. DuPont Co.

7. A. P. Piechowski and G. R. Bird, Optics Comm. 50, 386 (1984),

8. A. V. Aristov and Yu S. Maslyukov, Optics and Spectroscopy 32, 181 (1972). English translation.

9. P. R. Hammond, J. Photochem. 10, 467 (1979).

10. R. R. Sauers, S. N. Husain, A. P. Piechowski and G. R. Bird, submitted to Liebig's Annalen der Chemie.

11. D. L. Morel, E. L. Stogryn, A. K. Ghosh, T. Feng, P. E. Purwin, R. F. Shaw, C. Fishman, G. R. Bird and A. P. Piechowski, J. Phys. Chem. 88, 923 (1984).

12. A. P. Piechowski, G. R. Bird, D. L. Morel and E. L. Stogryn, J. Phys. Chem. 88, 934 (1984).

13. L. G. S. Brooker, G. H. Keyes, R. H. Sprague, R. H. Van Dyke, E. Van Lare, G. Van Zandt, F. L. White, H. W. J. Cressman and S. G. Dent, Jr., J. Am. Chem. Soc. 73, 5332 (1951). This is the tenth paper in the "Colour and Constitution" series, all in this journal.

14. J. R. Platt, J. Chem. Phys. 25, 80 (1956).

15. J. R. Platt, J. Chem. Phys. 34, 862 (1961).

16. M. Sadrai and G. R. Bird, Opt. Commun. 51, 62 (1984).

17. S. M. Grenci, Fluorescence Efficiency of Several Cyclized Merocyanine and Azamerocyanine Dyes, doctoral thesis, Rutgers University, 1983.

18. M. Koizumi, S. Kato, N. Mataga, T. Matsuura and Y. Usai, Photosensitized Reactions (Kagakudojin Publishing Co., Kyoto, Japan, 1978), text in English; see especially pp. 155-160.

19. P. M. Felker, W. R. Lambert and A. H. Zewail, J. Chem. Phys. 77, 1603 (1982), and references therein.

20. A. V. Buettner, B. B. Snavely and O. G. Peterson, Proc. Int'l. Conf. on Mol. Lum. (W. A. Benjamin, New York, 1969), p. 403.

21. J. P. Webb, W. C. McColgin, O. G. Peterson, D. L. Stockman and J. H. Eberly, J. Chem. Phys. 53, 4227 (1970).

22. G. A. Ketsle, L. V. Levshin, T. D. Slavnova and A. K. Chibisov, Soviet Physics (Doklady) 16, 986 (1972), English translation.

23. K. Kato, Optics Lett. 9, 544 (1984). 